\title{
Ecología humana y la urbe inteligente: Utilizando mapeo interactivo para el análisis socio-ambiental del uso de agua y de energía eléctrica en lbarra-Ecuador
}

\section{Human ecology and the Smart city: using interactive maping for the socioenvironmental analysis of water and electricity use in Ibarra - Ecuador}

Larry M Frolich, Ph.D. Investigador Prometeo, Universidad Técnica del Norte, Av 17 de Julio 5-21, Ibarra Docente Ciencias Naturales, Universidad de Miami Dade. Ifrolich@mdc.edu

Jorge Adrian Caraguay Procel, Profesor Principal, Universidad Técnica del Norte, Facultad Ingeniería en Ciencias Aplicadas. Av 17 de Julio 5-21, Ibarra jacaraguay@utn.edu.ec

Oscar Rosales, Profesor, Universidad Técnica del Norte, Facultad Ingeniería en Ciencias Agropecuarias y Ambientales, oscar id2003@yahoo.es

Andres Cardenas, Ing., Maestrante Ingeniería de Software, Universidad Técnica del Norte. Av 17 de Julio 5-21, Ibarra.afcardenasp@utn.edu.ec

Patricio Castro Ing. en Sistemas Computacionales, Estudiante de Postgrado Maestría de Software, Universidad Técnica del Norte. hpcastro@utn.edu.ec

Patricia Isabel Llanga Ochoa, Estudiante de Maestría de Gestión Sustentable de Recursos Naturales, Instituto de Postgrado, Universidad Técnica del Norte, IbarraEcuador. isabel Ilanga@hotmail.com

Ana María Lucero, Ing., Estudiante de Maestría de Gestión Integral de Cuencas Hidrográficas, Instituto de Postgrado, Universidad Técnica del Norte. Av 17 de Julio 5-21, Ibarra luceroanamaria7@gmail.com

Fernando Rea, Ing. Sistemas Computacionales, Estudiante de Maestría en Ingeniería de Software, Universidad Técnica del Norte. Av 17 de Julio 5-21, Ibarra rfreae@utn.edu.ec

Alexandra Elizabeth Jácome Ortega, Docente FICAYA, Universidad Técnica del Norte. Av 17 de Julio 5-21, Ibarra ajacome@utn.edu.ec 


\title{
Esmeralda Guevara, Ph.D. Docente Distrito Miami Dade. esmeche@yahoo.com
}

Fausto Sarmiento, Ph.D. Docente Geografia, Universidad de Georgia, fsarmien@uga.edu

\section{RESUMEN}

Se desarrolla una interface GIS, disponible para técnicos y público en general, en la que se muestra el análisis sectorial de los usuarios de agua potable y energía eléctrica. Se utiliza herramientas de BI (Business Intelligence), con el fin de realizar la analítica de los datos que se generan a diario en las empresas proveedoras de los servicios, esto permite presentar dinámicamente los cambios en uso del recurso por zona y en el tiempo. La investigación, reúne a profesionales de diferentes áreas, convirtiéndolo en un trabajo inter disciplinario, que incluyen datos socio-económicos del último censo del país y datos ambientales del catastro urbano, para identificar los factores claves que influyen en el consumo del recurso. Con la facilidad de visualizar geográficamente y sobre el tiempo con datos reales y actuales del uso de agua y energía eléctrica comparado con factores socio-ambientales, se simplifica las decisiones entre todas las instituciones e individuos involucrados. Así, con macro datos constantemente actualizados y presentados de manera fácil para interpretar, se logra un paso hacia una urbe inteligente que contribuya a la calidad de vida. A la vez, mientras se aplica las tecnologías avanzadas a los datos actuales el uso y análisis debería ocurrir dentro de un marco histórico, reconociendo la cultura, el ambiente, y el deseo de aumentar el Buen Vivir para todos los residentes de la zona.

\section{Palabras claves:}

Urbe inteligente; Ecología Humana; Mapeo Interactivo; Recursos Urbanos; IbarraEcuador

\begin{abstract}
Analysis of the tens of thousands of users of drinking water and electricity in the city of Ibarra, Ecuador will be presented in an online interactive map available to utility technical analysts as well as the public at large. We will use $\mathrm{BI}$ (Business Intelligence) tools to present temporal and geographic dynamics in resource use. Our interdisciplinary team also applies the tools of socio-ecological analysis to incorporate household economic data from the national census and environmental data from the city property records to identify key factors that influence resource use. The ease of visualizing, geographically and temporally with real-time data, the use of water and electricity, overlaid by socio-ecological
\end{abstract}


factors, will simplify decisions among all of the stakeholder institutions and individuals. Real-time big data made available and presented in an easy-tointerpret format represents a big step towards "smart-city" planning in favor of quality of life for all. At the same time, although using advanced technology to interpret complex data, we work within a historical context that recognizes the culture, environment and desire to augment "El Buen Vivir" for all residents of the region.

\section{Key words}

Smart City; Human Ecology; Interactive Map; Urban Resources; Ibarra, Ecuador

\section{INTRODUCCIÓN}

La disponibilidad de dos recursos-agua potable y energía eléctrica-son características fundamentales de una zona urbana bien desarrollada (Sahely et al., 2005; Zygiaris, 2013). A la vez, su ausencia tienda a definir zonas marginales y barrios sub-desarrollados (Estache et al., 2001; Komives, 2005). En el siglo XXI, se está aplicando herramientas de análisis digital, a través de sistemas de computación de alto poder, para realizar una planificación integral que incorpore múltiples elementos que afecte el suministro y la demanda de estos dos recursos, así formando parte de lo que se denomina una urbe inteligente. Aunque es fácil crear y administrar tales sistemas de análisis como herramienta puramente técnica, es importante incorporar un entendimiento del entorno socio-ambiental, o lo que se refiere como la "ecología humana urbana" (Shulenberger et al., 2008)

El Proyecto Ibarra Verde pretende crear una herramienta de análisis, basado en un mapeo integral de la zona urbana de la ciudad de Ibarra, en los Andes Norte del Ecuador, que sirva como modelo para una planificación urbana técnicamente alta, y que, a la vez, respete el sentido cultural, las normas sociales y el estado ambiental de la región. Por un lado, aquí presentamos los retos y avances en la formación de la herramienta en sí, desde el punto de vista estrictamente técnica. Pero también tomamos en cuenta unos supuestos del desarrollo urbano, con procedencia de la cultura occidental que no siempre aplique en nuestro entorno, notando que estos mismos supuestos tal vez ni son favorables para calidad de vida en el hemisferio Norte. En este sentido pretendemos ofrecer no solamente una opción regional para el desarrollo urbano en favor de calidad de vida, o el "buen vivir," sino un punto de referencia que sirva como modelo a nivel mundial.

El reto de crear herramientas digitales de alta calidad para analizar el uso de recursos en la zona urbana es una respuesta lógica al crecimiento de la ciudad, tal fenómeno que define la evolución del ser humano, como especie global (de tal punto que se ha denominado una nueva especie Homo evolutis-Gullans \& Enriquez, 2011), en nuestros tiempos. Durante el siglo XX, la mitad de la población humana ha migrado a las áreas urbanas, principalmente en el hemisferio norte (Cohen, 2003; Hall, 2014; Zimmerman, 1926). La Organización de las Naciones Unidas (ONU) ha determinado que en el año 2013 se llegó al punto de inflexión en que la mayoría de la población mundial vive en ciudades (UN-DESA, 2014). Las ventajas del ámbito urbano son numerosas, no solamente por el deseo del vibrante distrito central, sea cultural, bancario o industrial, (Carrión, 2005) sino 
incluso por las economías de escala, facilidad de provisionar necesidades vitales, la concentración de diversidad humana, la posibilidad de fomentar innovación y fácil acceso a nuevas tecnologías (Johnson, 2006). A la vez, la migración al ámbito urbano presenta el riesgo de una disminución de la calidad de vida, hasta niveles de miseria entre el segmento pobre de la población (Mingione, 1996; Wratten, 1995). Este proceso de degradación en las áreas urbanas ocurre cuando se pierde el acoplamiento entre el ser humano y la realidad biogeográfica de la zona (Satterhwaite, 2003).

En el caso de la ciudad de Ibarra-Ecuador, encontramos una situación ideal para la creación de una herramienta técnicamente alta para guiar el desarrollo de la zona. En los últimos dos décadas, el nivel técnico de planificación de las entidades públicas principales, entre ellos el gobierno municipal, la empresa municipal de agua potable, y la empresa regional de energía eléctrica, se ha aumentado al punto de tener disponibles cantidades de datos digitales sobre múltiples aspectos de la vida urbana. Datos disponibles incluyen el uso de agua y energía de cada hogar sobre los últimos cinco años, una base catastral completamente digitalizada y datos del censo sobre la situación socio-económico de las familias de la región. Pero no se encuentra todavía una centralización de análisis que incorporen estos múltiples elementos para guiar los técnicos, igual que el público, en la inevitable expansión de la zona urbana sobre el transcurso de este siglo. Entonces existe una oportunidad grande de ajuntar estos varios componentes de la calidad de vida bajo un solo techo, en forma de una herramienta interactiva de último modo. Pero a la vez, se presenta unos retos para sobrepasar incluso un severo límite de recursos para crear una herramienta centralizada de planificación, resistencia de compartir datos de parte de las entidades involucradas y la necesidad de unir datos en una sola base para poder manipularlos y analizarlos en favor de un sistema unificado. ¿Entonces, dado estos retos igual que oportunidades, como desarrollamos una herramienta técnicamente alta que aproveche de la riqueza de datos mientras supere la escasez de recursos técnicos y que también respete las normas culturo-socioambientales de la región? Como delineamos en las metodologías abajo estamos enfrentando y sobrepasando estos retos, pero siempre con un ojo a la necesidad de respetar el ámbito cultural en que trabajamos.

\section{MÉTODO}

\subsection{Datos de agua potable y energía eléctrica}

En colaboración con las empresas públicas de agua potable (EMAPA-IEmpresa Municipal de Agua Potable y Alcantarillado-lbarra) y la empresa pública de energía eléctrica (EmelNorte-Empresa Eléctrica Regional Norte), hemos conseguido datos del uso de agua potable y de energía eléctrica, para cada usuario doméstico igual que industrial, sobre los últimos cinco años, debidamente digitalizados. Como parte del análisis de datos, estamos incorporando la ubicación geográfica, con coordenadas precisas de cada usuario para poder analizar distribución de uso por sectores geográficos y tendencias históricas del uso de agua y de energía en un mapeo integrado e interactivo.

Para analizar las tendencias temporales y la distribución geográfica del uso de estos dos recursos, los datos se unificarán en una base de Oracle para 
aplicar las herramientas de Oracle $\mathrm{BI}$ (Business Intelligence) una poderosa herramienta basada en tecnología Web con interfaz gráfica amigable para los usuarios y que nos permite reunir todos los datos y transformarlos en información que podrá servir para toma de decisiones. Para proteger la privacidad del ciudadano, estos datos se presentarán como promedios dentro de cada zona censal.

\subsection{Datos socio-ambientales}

De procedencia de la entidad de apoyo social SENPLADES (2014), tenemos disponibilidad de información sobre la situación socio-económica de los hogares de la región en base al censo poblacional 2010, sectorizado por zonas censales amanzanados o dispersos abarcando aproximadamente 400 personas. Estos datos revelan áreas censales en kilómetros cuadrados, número de habitantes, densidad de la población por zona censal, número de viviendas, número de hogares, población económicamente activa (PEA) e índice social de necesidades básicas insatisfechas (NBI).

\subsection{Datos catastrales}

En colaboración con la entidad gubernamental municipal, tenemos acceso a la base catastral de la región, tal información que nos ayude incluir promedios de la superficie de los predios y del área de construcción que abarca cada hogar de la región. Para proteger la privacidad del ciudadano, estos datos también se presentarán como promedios dentro de cada zona censal.

Para poder correlacionar, por zonas y sobre tiempo, el uso de cada recurso con la situación promedio de la zona de censo poblacional, se presentarán los datos en forma interactiva en un sistema de mapeo disponible online en el browser, mediante la utilización de Geoserver (Servidor de mapas) y un visor geográfico que permitirá incorporar las capas del mapeo trabajadas en la herramienta digital ArcGIS y toda la consulta que se haga en el visor geográfico permitirá incorporar los datos en el Oracle $\mathrm{BI}$ para el análisis estadístico y gráfico del uso de los recursos. A la vez, el uso de recurso se comparará, en pantalla interactiva, con los datos socio-ambientales de los hogares de la región. sistema permitirá analizar la situación actual igual que las tendencias históricas.

\section{RESULTADOS}

\subsection{Tendencias y distribución del uso de agua y energía eléctrica}

Se revela una distribución de carácter estadística "normal" para el uso de agua en toda la ciudad igual por cada sector, facilitando un análisis a través de promedios y desviaciones estándar; aunque es importante notar un gran número de usuarios sin registrar uso ninguno o solo de un metro cubico, lo que representaría medidores nuevos o fuera de servicio, y no se los consideran en el análisis (Figure 1). 


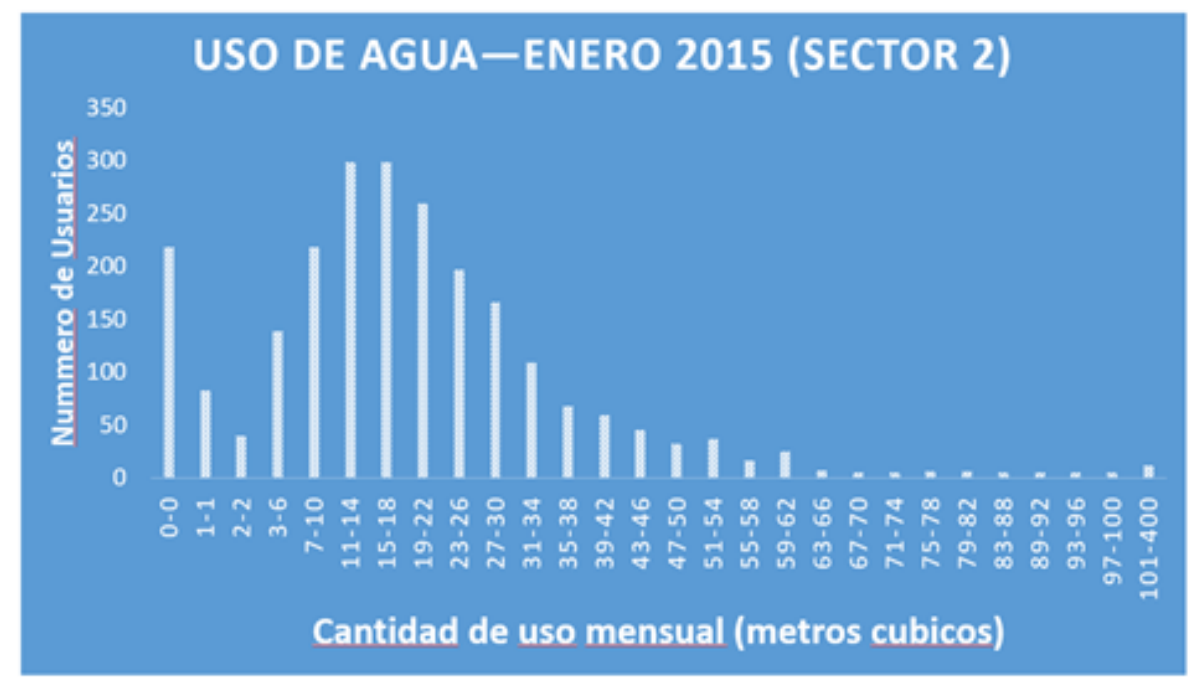

Figura 1 Ejemplo de la distribución normal del uso de agua, con un promedio de aproximadamente 12 metros cúbicos por mes, en este caso por Sector 2 de la empresa municipal del agua. Los otros sectores, igual que toda la zona urbana tiene la misma distribución normal, pero los promedios y la desviación varían entre sectores.

El uso de agua se ha aumentado dramáticamente para toda la región sobre los últimos cinco años (Figura 1).

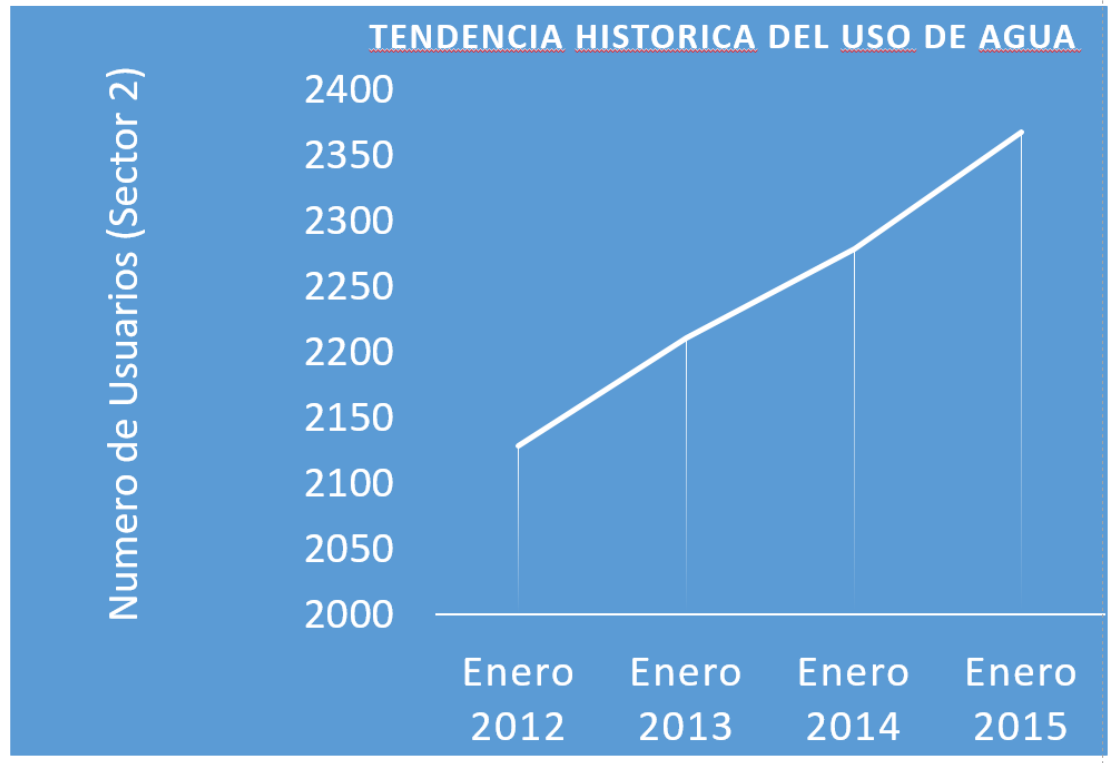

Figura 2 Ejemplo del crecimiento del uso de agua sobre los últimos 5 años. La zona urbana total muestra así mismo un incremento de aproximadamente 12 por ciento en número de usuarios. 
En el caso de la energía eléctrica, el consumo en kw/h de los abonados residenciales del cantón Ibarra indica un aumento impresionante durante los 3 últimos años (Figura 3).

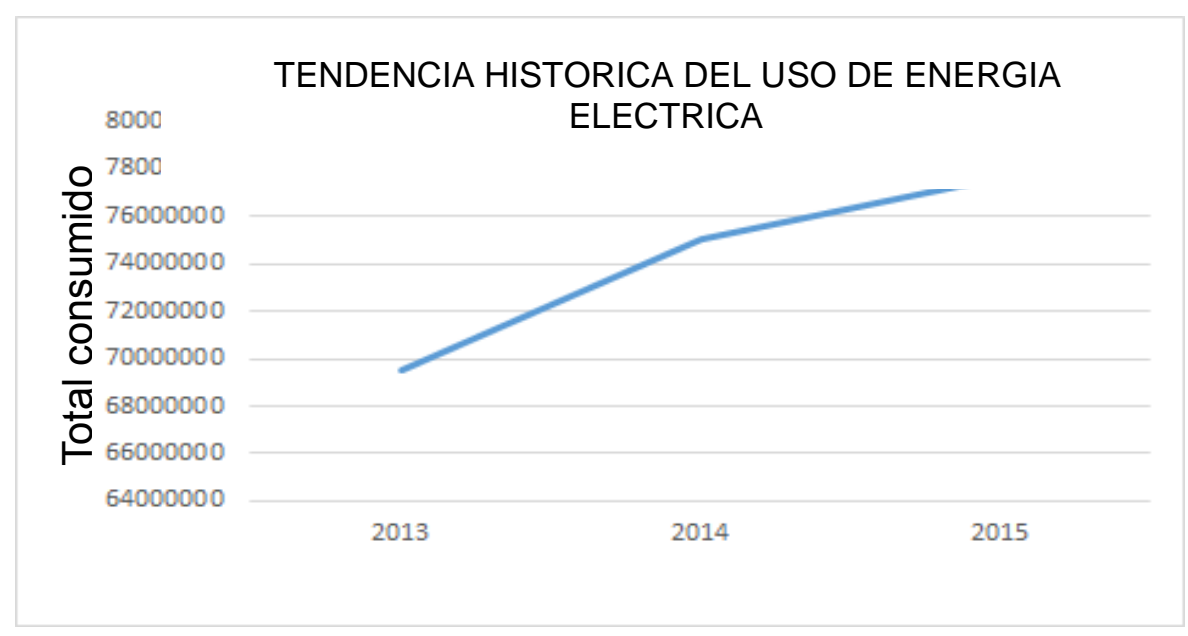

Figura 3 Ejemplo de incremento de uso de energía eléctrica sobre los últimos tres años por todo al Cantón Ibarra indicando un incremento de aproximadamente 11 por ciento en el uso total de electricidad.

\subsection{Mapeo a través de zonas censales}

Hemos adoptado las mismas zonas que delimite el censo nacional para facilitar comparación entre sectores del mismo tamaño, dado que cada sector abarca aproximadamente 400 personas. El mapa de las zonas censales (Figura 4 ) indica la concentración de la población urbana en el centro de la ciudad, por el tamaño geográfico reducido de estos sectores, comparando con los barrios alrededores a donde la población es menos densa y las zonas censales son más alargadas.

\subsection{Distribución geográfica de factores socio-ambientales}

La superficie de los predios y el área de construcción se encuentra, en general en opuesto, con superficies limitados, pero porcentaje de construcción alto en el centro de la ciudad, mientras los barrios alrededores con superficie más grande, pero con porcentaje de construcción limitada (Figure 5).

\subsection{Sistema interactivo de mapeo}

Para poder interactuar con los datos con el afán de que cualquier usuario del sistema cree su proprio análisis de la relación entre el uso de recurso y los factores socio-ambientales que le interese, el sistema presenta una pantalla a donde los datos aparecen a través de los sectores y los factores que escoja el usuario (Figura 6). 


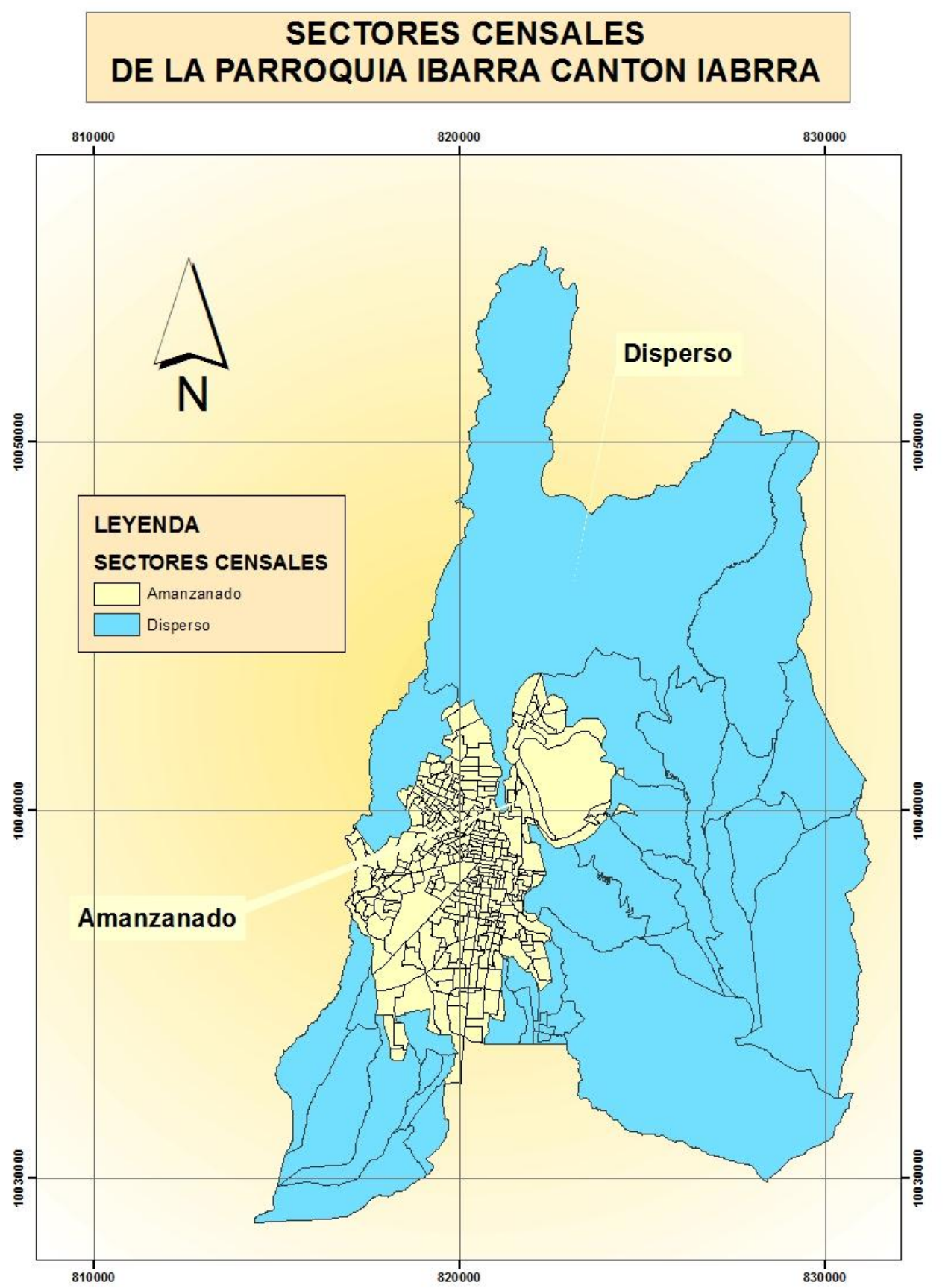

Figura 4 Mapa de sectores censales del Cantón lbarra. Cada sector abarca aproximadamente 400 personas, así la región "amanzanado" incluye la parte urbana con sectores geográficamente pequeñas y con alta densidad de población, mientras la región "dispersa" incluye áreas rurales con sectores geográficamente grande y baja densidad de población (fuente: SENPLADES, 2014). 


\section{DISCUSIÓN}

Dos dualidades falsas poderosamente guían a veces sub-conscientemente nuestros esfuerzos de crear ámbitos urbanos vivibles (Frolich et al., 2015). La dualidad de humano-natural tiene raíces en la cultura judeo-cristiana desde la narrativa de la creación de la Biblia, y está desarrollado como parte integral de la cultura occidental. De esta dualidad viene el sentido de una lucha entre lo que hace el ser humano y lo que pasa en el mundo "natural," entendido como un mundo aparte (Dove \& Kammen, 2015; Glacken, 1967). La mezcla cultural mestizo de las Américas todavía no decide si acepta la falsa dualidad entre ser humano y lo natural con procedencia occidental, o un sentido aboriginal adonde el ser humano vive cerca a la "pacha mama." Hasta no aceptar que el mito de lo "prístino" ha sido ya desmitificado por los geógrafos (Denevan, 2011) y ecólogos (Balée, 2014) contemporáneos, para una zona urbana en comparación a un parque nacional no avanzaremos en proveer mejor calidad de vida a la gran demografía urbanizada del siglo XXI.

La dualidad de urbano-rural es tal vez primordial pero también engañador. Por un lado, es obvio que la concentración de población en una ciudad crea un estilo de vida sumamente distinta de lo de la zona agrícola o rural. Pero es importante entender que la ciudad todavía incorpore los mismos suelos, agua, aire y energía que existe en una zona menos poblada. En este sentido, se debería aplicar los entendimientos del uso de suelo, de la cuenca hidrográfica y del flujo de energía con su concomitante potencialidad de contaminar el aire que se aplica en cualquier parte. Pero el rato que el agua esta entubado y el suelo está cubierto por cemento, encontramos con la tendencia de subestimar su efecto a la calidad de vida del ser humano. Unas tendencias de la planificación urbana incluyen la incorporación de jardines caseros y áreas de finca dentro de la zona poblada, y el exponer del agua potable y agua no potable en riachuelos, zanjas y espacios públicos a donde el agua forma parte del paisaje urbano (Pickett et al. 2008).

El efecto de la urbanización sobre la vida del ser humano abarca casi cada aspecto de la vida desde el inicio del Época Antropoceno. A nivel del individuo, de la familia, y de la sociedad, cada aspecto de la vida humana cambia en el ámbito urbano. En el caso del agua, la presencia de una red de distribución de agua entubada y alcantarillas para aguas servidas, aunque no siempre presente en zonas urbanas y a veces disponibles en áreas rurales, es un sello de distinción de las zonas urbanas. La red implica el desarrollo de fuentes seguras de agua, plantas de tratamiento, sistemas de regulación de la distribución, y plantas de tratamiento de las aguas servidas. Con la red de agua, la potencialidad de controlar las enfermedades contagiosas es alta, pero el riesgo de disponer agua contaminada también está presente. Mientras la gente se alivia del trabajo diario de traer agua se pierde el conocimiento de la fuente del agua y el sentido de ver el agua directamente en un río o una laguna.

En el caso del uso de agua y energía, el análisis pretende incorporar una vista hacia la totalidad del uso y su relación con el diseño de la ciudad y la situación de la población de cada zona. Hay una tendencia, de procedencia del hemisferio Norte, pero con reconocimiento de los tiempos ancestrales, a incorporar áreas de jardín, de bosque, de pajonal, y de agricultura dentro de la ciudad, todo relacionado, por supuesto, con la distribución del agua y el sistema cultural de los residentes. Es importante, entonces, poder comparar, interactivamente, la 
situación demográfica, económica, social y ambiental de cada zona para incorporar dentro de las sugerencias de planificación en referencia a la distribución y suministro del recurso agua.

En el caso de la energía, una red intensa de distribución de luz eléctrica provee la mayoría de las necesidades de energía en la zona urbana, incluso últimamente la energía para cocinar con estufas eléctricas. En vez de transportarse a pie o a caballo, la gente en la zona urbana depende del uso de vehículos con motores de combustible fósil, sea diésel, gasolina o, como vamos a ver con más frecuencia en el futuro cercano, a través de electricidad. Para calentar las casas, poco se utiliza ya la madera o el carbón, como es común en una zona rural, sino se usan instalaciones de gas o energía eléctrica; y con frecuencia en zonas cálidas, se enfría las casas con aire acondicionado con el uso de luz eléctrica. Así, igual con el agua, la habilidad de comparar el uso de energía eléctrica con la situación socio-ambiental de cada zona facilitará diseños mejores en referencia a la calidad de vida y el uso de energía eléctrica.

Una urbe inteligente en el siglo XXI tendrá que realizar un análisis continuo, aprovechando de bases grandes de datos, como estamos creando con el Proyecto Ibarra Verde, para asegurar una planificación equitativa que respete las normas culturales, los índices sociales y la situación ambiental de la ciudad. 


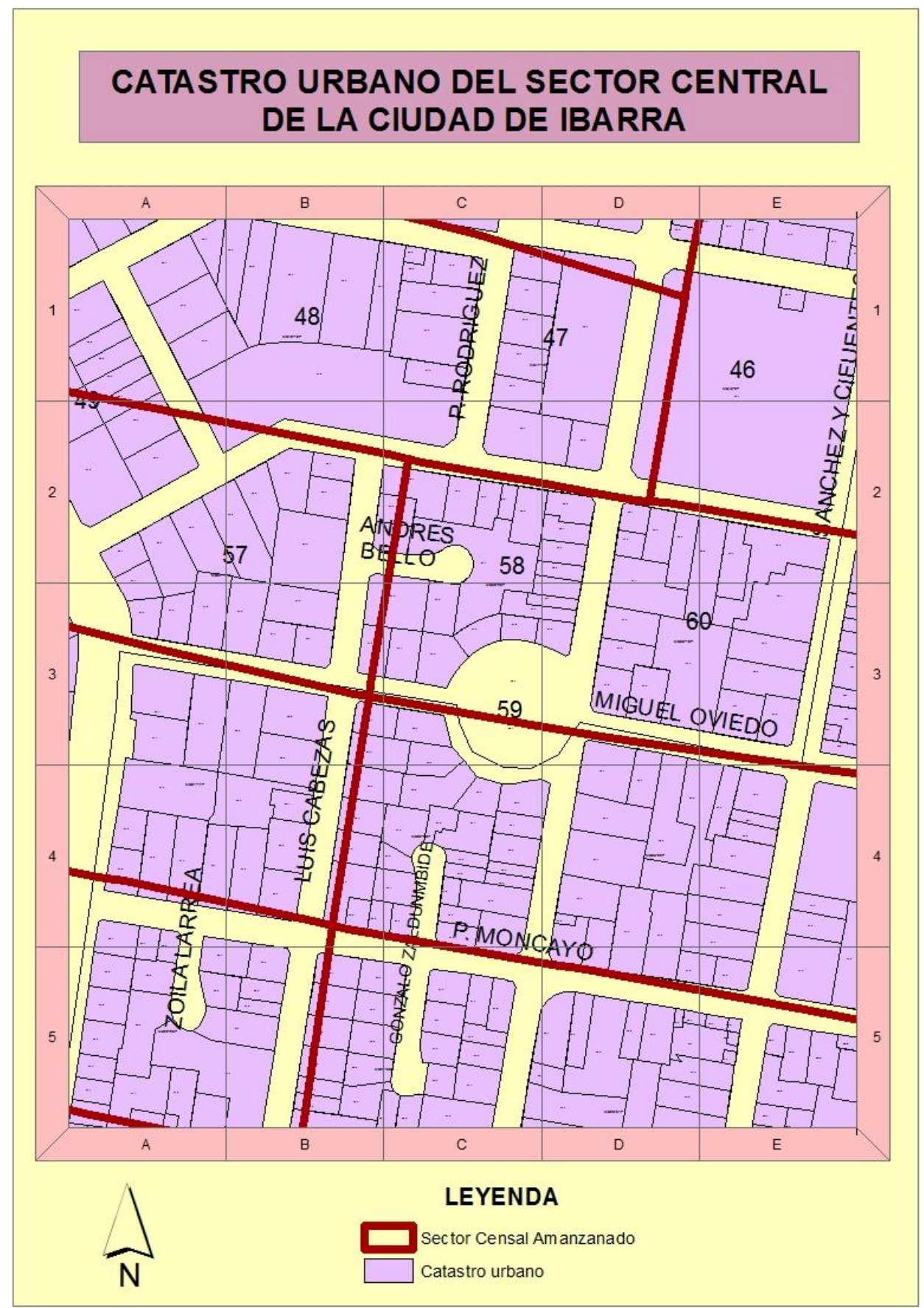

Figura 5 Ejemplo de mapeo catastral municipal de Ibarra indicando el tamaño de predios. El área indicada es del centro de la ciudad adonde los predios son pequeños, pero altamente construida. El área de construcción para cada predio está disponible a través de la base de datos municipal. 


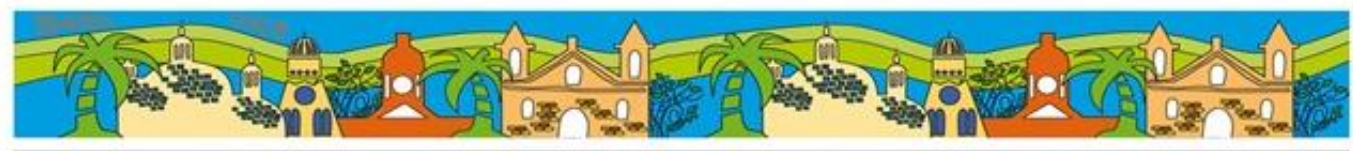

Sistema integrado de datos socio-ambientales para análisis y planificación urbana

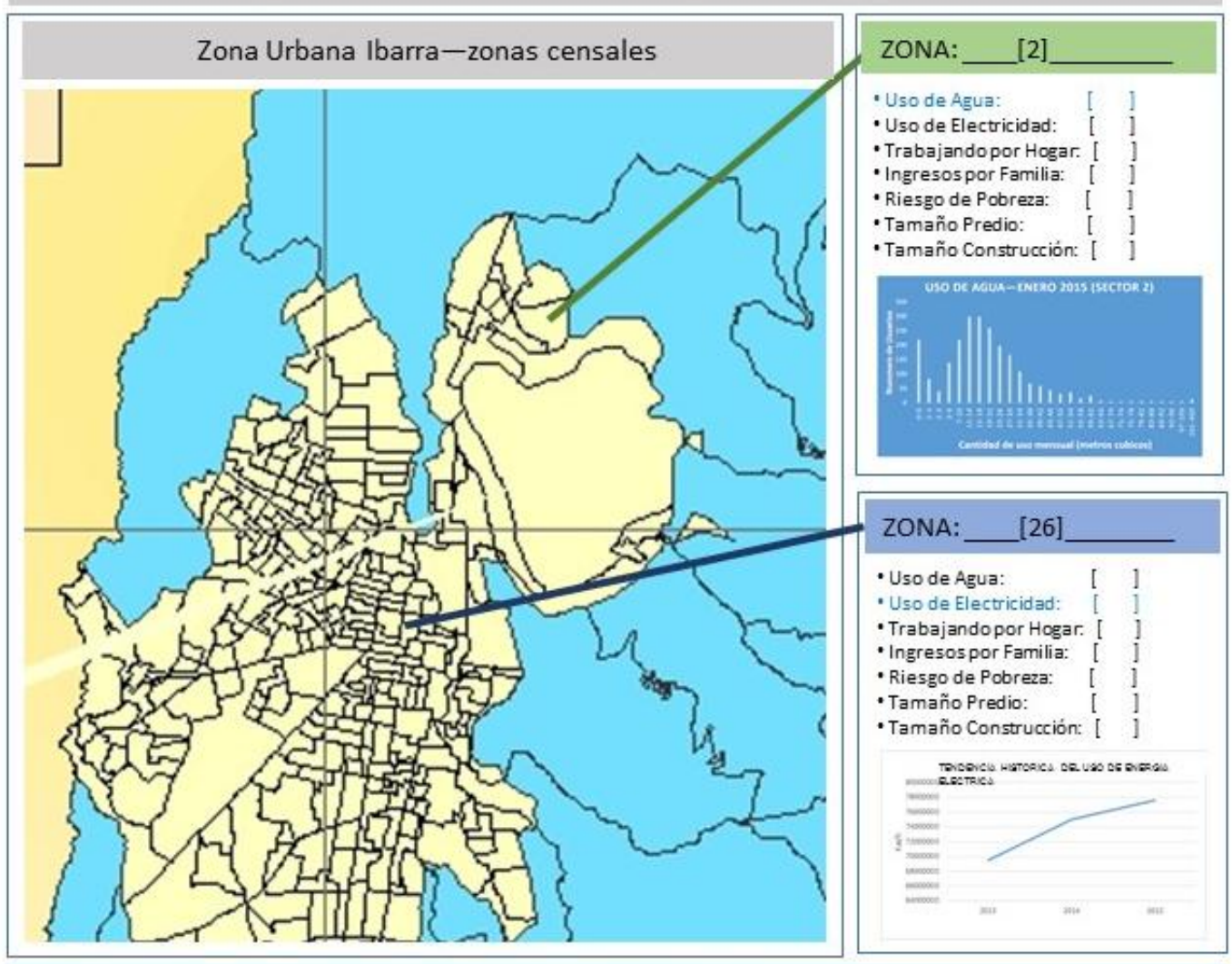

\section{Sección Estadistica}

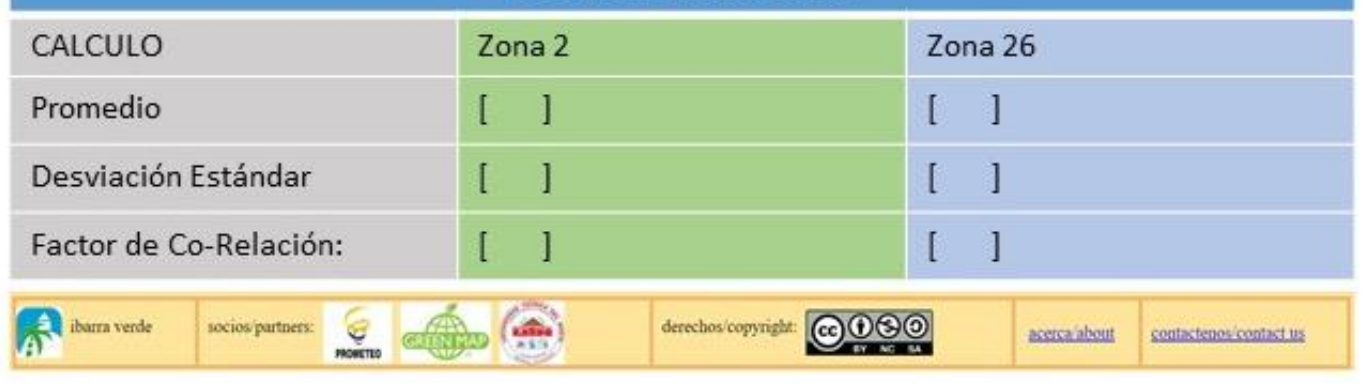

Figura 6 Bosquejo de página principal del sitio lbarra Verde indicando mapeo interactivo con dos sectores (2 y 26) escogidos como ejemplares para comparar datos básicos, gráficos elaborados e información estadística de cada sector. Nota que los gráficos no son datos definidos sino ejemplos de la interactividad del sitio. 


\section{REFERENCIAS}

1 Balée, W., (2014). " Historical Ecology and the Explanation of Diversity: Amazonian Case Studies." In: Applied Ecology and Human Dimensions in Biological Conservation. New York: Springer

2 Carrión, F., 2005. "The Historic Center as an Object of Desire." In: Carrión, F. and L.M Hanley (editors). Urban Regeneration and Revitalization in the Americas: Towards a Stable State. The Wilson Center.

3 Denevan, W.M., (2011). "The Pristine Myth Revisited." The Geographical Review 101(4): 576-591.

4 Dove, M.R. \& D. Kammen, (2015). "Science, Society and the Environment: Applying Anthropology and Physics to Sustainability." Oxford: Routledge.

5 Glacken, C.J., (1967). " Traces on the Rhodian Shore: Nature and Culture in Western Thought from Ancient Times to the End of the Eighteenth Century." Berkeley: University of California Press, 1967.

6 Gullans, S., \& Enriquez, J., (2011). "Homo evolutis." TED Books.

7 Estache, A., Gomez-Lobo, A., \& Leipziger, D., (2001). "Utilities privatization and the poor: lessons and evidence from Latin America." World Development, 29(7), 1179-1198.

8 Johnson, S., (2006). "The Ghost Map: The Story of London's Most Terrifying Epidemic--and How It Changed Science, Cities, and the Modern World." New York: Penguin.

9 Komives, K., (2005). "Water, electricity, and the poor: Who benefits from utility subsidies?." World Bank Publications.

10 Mingione, E., (1996). "Urban Poverty and the Underclass." Cambridge, MA: Blackwell Publishers. Pickett, S. T., Cadenasso, M. L., Grove, J. M., Nilon, C. H., Pouyat, R. V., Zipperer, W. C., \& Costanza, R. (2008). Urban ecological systems: linking terrestrial ecological, physical, and socioeconomic components of metropolitan areas. In Urban Ecology (pp. 99-122). Springer US.

11 Sahely, H. R., Kennedy, C. A., \& Adams, B. J., (2005)." Developing sustainability criteria for urban infrastructure systems." Canadian Journal of Civil Engineering, 32(1), 72-85.

12 Satterthwaite, D., (2003)." The Links between Poverty and the Environment in Urban Areas of Africa, Asia, and Latin America." The ANNALS of the American Academy of Political and Social Science. vol. 590 no. 1: 73-92

13 SENPLADES (2014). Cartografía digital sectores censales de Imbabura.

14 Shulenberger, E., Endlicher, W., Bradley, G., Ryan, C., ZumBrunnen, C., \& Simon, U., (2008)." Urban ecology: an international perspective on the interaction between humans and nature." J. Marzluff (Ed.). Springer Science \& Business Media.

15 Wratten, E., (1995). “ Conceptualizing urban poverty." Environment and Urbanization. vol. 7 no. 1: 11-38.

16 UN-DESA., (2014). "World Urbanization Prospects." Report from the United Nations Population Division. New York, NY. 
17 Zygiaris, S., (2013)." Smart city reference model: Assisting planners to conceptualize the building of smart city innovation ecosystems." Journal of the Knowledge Economy, 4(2), 217-231. 